\title{
Assimilation of remote sensing observations into a continuous distributed hydrological model: impacts on the hydrologic cycle
}

\author{
Paola Laiolo (1), Simone Gabellani (1), Lorenzo Campo (1), Luca Cenci (1,3), Francesco Silvestro (1), Fabio \\ Delogu (1), Giorgio Boni (1,2), and Roberto Rudari (1) \\ (1) CIMA Research Foundation, Savona, Italy, (3) WRR Programme, UME School, IUSS-Pavia, Italy, (2) DIBRIS, University \\ of Genova, Italy
}

The reliable estimation of hydrological variables (e.g. soil moisture, evapotranspiration, surface temperature) in space and time is of fundamental importance in operational hydrology to improve the forecast of the rainfall-runoff response of catchments and, consequently, flood predictions. Nowadays remote sensing can offer a chance to provide good space-time estimates of several hydrological variables and then improve hydrological model performances especially in environments with scarce in-situ data. This work investigates the impact of the assimilation of different remote sensing products on the hydrological cycle by using a continuous physically based distributed hydrological model. Three soil moisture products derived by ASCAT (Advanced SCATterometer) are used to update the model state variables. The satellite-derived products are assimilated into the hydrological model using different assimilation techniques: a simple nudging and the Ensemble Kalman Filter. Moreover two assimilation strategies are evaluated to assess the impact of assimilating the satellite products at model spatial resolution or at the satellite scale. The experiments are carried out for three Italian catchments on multi year period. The benefits on the model predictions of discharge, LST, evapotranspiration and soil moisture dynamics are tested and discussed. 